\title{
NOTE ON SCHWARZ-PICK ESTIMATES FOR BOUNDED AND POSITIVE REAL PART ANALYTIC FUNCTIONS
}

\author{
SHAOYU DAI AND YIFEI PAN \\ (Communicated by Joseph A. Ball) \\ Dedicated to Professor Hu Ke on the occasion of his eighty-sixth birthday
}

ABStRaCT. In this note we consider higher order derivatives of bounded analytic functions.

\section{INTRODUCTION}

Let $\mathbb{D}$ be the unit disk in $\mathbb{C}$. If $\varphi(z)$ is a analytic function in $\mathbb{D}$, and $|\varphi(z)|<1$ for each $z \in \mathbb{D}$, the Schwarz inequality is

$$
\left|\varphi^{\prime}(z)\right| \leq\left(1-|\varphi(z)|^{2}\right) /\left(1-|z|^{2}\right) .
$$

In this paper we obtain an estimate of higher order derivatives for bounded analytic functions and functions with positive real part.

Theorem 1.1. Let $\varphi(z)$ be an analytic function in $\mathbb{D}$ and $|\varphi(z)|<1$ for each $z \in \mathbb{D}$. Then

$$
\left|\varphi^{(n)}(z)\right| \leq \frac{n !\left(1-|\varphi(z)|^{2}\right)}{\left(1-|z|^{2}\right)^{n}}(1+|z|)^{n-1} .
$$

By the same method, we can prove a version of Theorem 1.1 for analytic functions with positive real parts.

Theorem 1.2. Let $f(z)$ be an analytic function in $\mathbb{D}$ and $\Re f(z)>0$ for each $z \in \mathbb{D}$. Then

$$
\left|f^{(n)}(z)\right| \leq \frac{2 n ! \Re f(z)}{\left(1-|z|^{2}\right)^{n}}(1+|z|)^{n-1} .
$$

The following result shows that the inequality in Theorem 1.2 is asymptotically sharp for each boundary point of the unit disk. On the other hand we don't know if this is true for Theorem 1.1.

Theorem 1.3. Let $f(z)$ be an analytic function in $\mathbb{D}$ and $\Re f(z)>0$ for each $z \in \mathbb{D}$. Let $r=|z|, z=r e^{i \theta}$. Then for each $\theta$,

$$
\lim _{r \rightarrow 1}(1-r)^{n+1}\left|f^{(n)}(z)\right|=2 n ! \lim _{r \rightarrow 1} \frac{1-r}{1+r} \Re f(z) .
$$

Received by the editors October 19, 2006 and, in revised form, January 2, 2007.

2000 Mathematics Subject Classification. Primary 30C80.

We thank the referee for his or her valuable comments to this paper. 
We would like to point out that Theorem 1.1 gives an explicit bound of Theorem 1 in [1] or Theorem 3 in [2]. On the other hand, Theorem 1.1 was proved in 1984 by the second author in [5] as a lemma for $n=2,3$ and the general case here applies the extension of the method in [5]. Here is a corollary to Theorem 1.1.

Corollary 1.1. Let $\varphi(z)$ be an analytic self-map of $\mathbb{D}$. Then

$$
\sup _{z \in \mathbb{D}} \frac{\left|\phi^{(n)}(z)\right|\left(1-|z|^{2}\right)^{n}}{1-|\phi(z)|^{2}} \leq n ! 2^{n-1} .
$$

This result will give an explicit bound for Theorem 6 in [2] where $C_{n}$ can be taken as $(n+1) ! 2^{n+2}$. Here is the result.

Corollary 1.2. Let $\phi(z)$ be an analytic self-map of $\mathbb{D}$. Let

$$
\tau_{\phi, n}(z)=\frac{\left|\phi^{(n)}(z)\right|\left(1-|z|^{2}\right)^{n}}{1-|\phi(z)|^{2}} .
$$

Then $\tau_{\phi, n}(z)$ is Lipschitz with respect to the pseudohyperbolic metric:

$$
\left|\tau_{\phi, n}(z)-\tau_{\phi, n}(w)\right| \leq(n+1) ! 2^{n+2} \rho(z, w)
$$

for any $z, w \in \mathbb{D}$, where $\rho$ is the pseudohyperbolic distance.

As pointed out in [2], this result has an application to closed-range composition operators on the Bloch space.

\section{Proof of Theorem 1.1}

The proof of Theorem 1.1 is based on the following Lemma, which should be well-known and for which we include a simple proof (see [4]).

Let $\mathcal{B}$ be the class of analytic functions $\varphi(z)$ in $\mathbb{D}$, and $|\varphi(z)|<1$ for each $z \in \mathbb{D}$.

Lemma 2.1. Let $\varphi(z) \in \mathcal{B}$, and $\varphi(z)=\sum_{n=0}^{\infty} a_{n} z^{n}$. Then we have $\left|a_{n}\right| \leq 1-\left|a_{0}\right|^{2}$ when $n \geq 1$.

Proof. Suppose $k \geq 1$ is an integer and let $\omega_{j}=e^{\frac{2 \pi i}{k} j}$.

It is well known that $\omega_{1}$ is a primitive root of unity and hence, $\sum_{j=1}^{k} \omega_{j}^{s}=0$ if $s \leq k-1$.

Let $f(z)=\frac{1}{k} \sum_{j=1}^{k} \varphi\left(\omega_{j} z\right)$. Then

$$
f(z)=a_{0}+a_{k} z^{k}+a_{2 k} z^{2 k}+\ldots+a_{n k} z^{n k}+\ldots
$$

Consider

$$
g(z)=\frac{f(z)-a_{0}}{1-\overline{a_{0}} f(z)}=b_{k} z^{k}+o\left(z^{k}\right)
$$

where $b_{k}=\frac{a_{k}}{1-\left|a_{0}\right|^{2}}$ by a simple calculation. If $g(z)=\sum_{n=0}^{\infty} b_{n} z^{n}$, and $g(z) \in \mathcal{B}$, then by the Cauchy estimate $\left|b_{k}\right| \leq 1$. So we have $\left|a_{n}\right| \leq 1-\left|a_{0}\right|^{2}$ when $n \geq 1$.

In the following we give the proof of Theorem 1.1.

Proof. If $\varphi(z) \in \mathcal{B}$, we consider

$$
F(z)=\varphi\left(\frac{z+\zeta}{1+\bar{\zeta} z}\right)=\sum_{v=0}^{\infty} c_{v} z^{v} \in \mathcal{B},
$$


where $c_{v}$ depends on $\zeta$. It follows that

$$
\begin{gathered}
\varphi(z)=F\left(\frac{z-\zeta}{1-\bar{\zeta} z}\right)=\sum_{v=0}^{\infty} c_{v}\left(\frac{z-\zeta}{1-\bar{\zeta} z}\right)^{v} \in \mathcal{B}, \\
\varphi^{(n)}(\zeta)=\left.\sum_{v=0}^{\infty} c_{v} \frac{d^{n}}{d z^{n}}\left(\frac{z-\zeta}{1-\bar{\zeta} z}\right)^{v}\right|_{z=\zeta}
\end{gathered}
$$

when $n \geq 1$.

For a fixed $v$, let $f(z)=(z-\zeta)^{v}$ and $g(z)=(1-\bar{\zeta} z)^{-v}$. Then the following facts are clear. If $v=0, \frac{d^{n}}{d z^{n}}(f g)=0$. If $v \geq 1$, then $f^{(k)}(\zeta)=k$ !, if $k=v$. Otherwise $f^{(k)}(\zeta)=0$.

Hence we need only compute $g^{(n-v)}(\zeta)$, for $n \geq v$. A simple computation shows that

Thus, for $v \geq 1$ and $n \geq v$,

$$
g^{(n-v)}(\zeta)=\bar{\zeta}^{n-v} \frac{v(v+1) \ldots(n-1)}{\left(1-|\zeta|^{2}\right)^{n}} .
$$

$$
\frac{d^{n}}{d z^{n}}(f g)(\zeta)=\sum_{k=0}^{n}\left(\begin{array}{l}
n \\
k
\end{array}\right) f^{(k)}(\zeta) g^{(n-k)}(\zeta)=\left(\begin{array}{l}
n \\
v
\end{array}\right) v ! g^{(n-v)}(\zeta) .
$$

Otherwise, $\frac{d^{n}}{d z^{n}}(f g)(\zeta)=0$.

Therefore,

$$
\varphi^{(n)}(\zeta)=\sum_{v=1}^{n} c_{v}\left(\begin{array}{l}
n \\
v
\end{array}\right) v ! g^{(n-v)}(\zeta)=\sum_{v=1}^{n} c_{v} \frac{(\bar{\zeta})^{n-v}}{\left(1-|\zeta|^{2}\right)^{n}} \frac{n !(n-1) !}{(n-v) !(v-1) !} .
$$

Note that $c_{0}=\varphi(\zeta)$, so by Lemma 2.1, we have when $v \geq 1$,

$$
\left|c_{v}\right| \leq 1-\left|c_{0}\right|^{2}=1-|\varphi(\zeta)|^{2} .
$$

Therefore,

$$
\begin{aligned}
\left|\varphi^{(n)}(\zeta)\right| & \leq \sum_{v=1}^{n}\left|c_{v}\right| \frac{|\zeta|^{n-v}}{\left(1-|\zeta|^{2}\right)^{n}} \frac{n !(n-1) !}{(n-v) !(v-1) !} \\
& \leq \frac{n !\left(1-|\varphi(\zeta)|^{2}\right)}{\left(1-|\zeta|^{2}\right)^{n}} \sum_{v=1}^{n} \frac{(n-1) !}{(n-v) !(v-1) !}|\zeta|^{n-v} \\
& =\frac{n !\left(1-|\varphi(\zeta)|^{2}\right)}{\left(1-|\zeta|^{2}\right)^{n}} \sum_{m=0}^{n-1} \frac{(n-1) !}{m !(n-m-1) !}|\zeta|^{m} \\
& =\frac{n !\left(1-|\varphi(\zeta)|^{2}\right)}{\left(1-|\zeta|^{2}\right)^{n}} \sum_{m=0}^{n-1}\left(\begin{array}{c}
n-1 \\
m
\end{array}\right)|\zeta|^{m} \\
& =\frac{n !\left(1-|\varphi(\zeta)|^{2}\right)}{\left(1-|\zeta|^{2}\right)^{n}}(1+|\zeta|)^{n-1} .
\end{aligned}
$$

Then replacing $\zeta$ by $z$ gives the desired result.

In the following, we use the Mobius transformation $f(z)=\frac{z+a}{1+\bar{a} z}$ to see how accurate the bound given in Theorem 1.1 is. When $n=1$, the equality holds in this case due to conformal invariance. But when $n \geq 2$, the equality does not hold even at the origin. But the estimate is sharp approximately. By calculation,

$$
\left|f^{(n)}(0)\right|=n !|a|^{n-1}\left(1-|a|^{2}\right) .
$$


There is the factor $|a|^{n-1}$ which occurs in the estimate. But we can see that when $a \rightarrow 1$, the estimation of $\left|f^{(n)}(0)\right|$ can be as good as one wants. It seems to be an interesting question: what is the best possible bound for Theorem 1.1?

\section{Derivatives for positive Real part analytic Functions}

If $f(z)=\sum_{n=0}^{\infty} c_{n} z^{n}$ with $\Re f(z)>0$ for each $z \in \mathbb{D}$, then we can prove Theorem 1.2 by using the well-known inequality $\left|c_{n}\right| \leq 2 \Re c_{0}$ (see [3]) and the exact same technique in proving Theorem 1.1. We omit the details here.

The estimation of Theorem 1.2 turns out best possible. Below we give a simple example.

Let $f(z)=\frac{1+z}{1-z}$. Obviously, $f(z)$ is an analytic function and $\Re f(z)>0$. By calculation, $f^{(n)}(z)=\frac{2 n !}{(1-z)^{n+1}}$. It follows that

$$
\left|f^{(n)}(z)\right|=\frac{2 n !}{|1-z|^{n+1}} .
$$

Therefore, when $z=x$, where $x$ is real number and $0 \leq x \leq 1$, we have $\left|f^{(n)}(x)\right|=$ $\frac{2 n !}{(1-x)^{n+1}}$.

On the other hand, by Theorem 1.3

$$
\frac{2 n ! \Re f(x)}{(1-|x|)^{n}} \frac{1}{1+|x|}=\frac{2 n ! \frac{1+x}{1-x}}{(1-|x|)^{n}} \frac{1}{1+|x|}=\frac{2 n !}{(1-x)^{n+1}} .
$$

Obviously, the equality in Theorem 1.1 holds when $z=x$.

From Theorem 1.3 we see that, if we let $r=|z|$, multiplying both sides of the estimates by $(1-r)^{n+1}$ and taking the limit as $r \rightarrow 1$ yields the equality. Namely, the estimation of Theorem 1.1 is asymptotically sharp for whole unit circle $|z|=1$.

Now we give the proof of Theorem 1.3.

Proof. We prove the case of $f(0)=1$ first.

Since $f(z)$ is an analytic function in $\mathbb{D}$ with $\Re f(z)>0$ for each $z \in \mathbb{D}$, and $f(0)=1$, then there is a unique positive Borel measure $\mu$ on $|z|=1$, such that

$$
f(z)=\int_{|\eta|=1} \frac{1+\eta z}{1-\eta z} d \mu(\eta), \int_{|\eta|=1} d \mu(\eta)=1 \quad \text { (see [3]). }
$$

By calculation,

$$
f^{(n)}(z)=2 n ! \int_{|\eta|=1} \frac{\eta^{n}}{(1-\eta z)^{n+1}} d \mu(\eta) .
$$

Let $z=r e^{i \theta_{0}}$. Then

$$
(1-r)^{n+1} f^{(n)}\left(r e^{i \theta_{0}}\right)=2 n ! \int_{|\eta|=1} \frac{\eta^{n}(1-r)^{n+1}}{(1-\eta z)^{n+1}} d \mu(\eta) .
$$

Consider $F_{\eta}(z)=\frac{(1-r)^{n+1}}{(1-\eta z)^{n+1}}$. Since $|z|<1,|1-\eta z| \geq 1-|\eta z|=1-|z|$, then $\left|F_{\eta}(z)\right| \leq 1$. Also,

$$
\lim _{r \rightarrow 1} F_{\eta}(z)= \begin{cases}1, & \eta=e^{-i \theta_{0}} \\ 0, & \eta \neq e^{-i \theta_{0}}\end{cases}
$$


Denote

$$
F_{0}\left(e^{i \theta_{0}}\right)= \begin{cases}1, & \eta=e^{-i \theta_{0}} \\ 0, & \eta \neq e^{-i \theta_{0}}\end{cases}
$$

By the Lebesgue Dominated Convergence Theorem

$$
\begin{gathered}
\lim _{r \rightarrow 1}(1-r)^{n+1} f^{(n)}\left(r e^{i \theta_{0}}\right)=2 n ! \int_{|\eta|=1} \lim _{r \rightarrow 1} \eta^{n} F_{\eta}(z) d \mu(\eta) \\
=2 n ! \int_{|\eta|=1} F_{0}\left(e^{i \theta_{0}}\right) \eta^{n} d \mu(\eta)=2 n ! e^{-i n \theta_{0}} \mu\left(\left\{e^{-i \theta_{0}}\right\}\right), \\
\lim _{r \rightarrow 1}(1-r)^{n+1}\left|f^{(n)}\left(r e^{i \theta_{0}}\right)\right|=\left|\lim _{r \rightarrow 1}(1-r)^{n+1} f^{(n)}\left(r e^{i \theta_{0}}\right)\right| \\
=\left|2 n ! e^{-i n \theta_{0}} \mu\left(\left\{e^{-i \theta_{0}}\right\}\right)\right|=2 n ! \mu\left(\left\{e^{-i \theta_{0}}\right\}\right) .
\end{gathered}
$$

On the other hand, based on the estimation we have $\left|f^{(n)}(z)\right| \leq \frac{2 n ! \Re f(z)}{(1-|z|)^{n}} \frac{1}{1+|z|}$. Multiplying $(1-r)^{n+1}$ to both sides above, it follows that

$$
(1-r)^{n+1}\left|f^{(n)}\left(r e^{i \theta_{0}}\right)\right| \leq 2 n ! \frac{1-r}{1+r} \Re f(z) .
$$

Considering $\frac{1-r}{1+r} \Re f(z)$, we have

$$
\Re f(z)=\int_{|\eta|=1} \frac{1-|z|^{2}}{|1-\eta z|^{2}} d \mu(\eta) .
$$

By the Lebesgue Dominated Convergence Theorem

$$
\lim _{r \rightarrow 1} \frac{1-r}{1+r} \Re f(z)=\int_{|\eta|=1} \lim _{r \rightarrow 1} \frac{(1-r)^{2}}{|1-\eta z|^{2}} d \mu(\eta)=\mu\left(\left\{e^{-i \theta_{0}}\right\}\right) .
$$

So

Hence

$$
\lim _{r \rightarrow 1} 2 n ! \frac{1-r}{1+r} \Re f(z)=2 n ! \mu\left(\left\{e^{-i \theta_{0}}\right\}\right) .
$$

$$
\lim _{r \rightarrow 1}(1-r)^{n+1}\left|f^{(n)}(z)\right|=\lim _{r \rightarrow 1} 2 n ! \frac{1-r}{1+r} \Re f(z) .
$$

Next we prove the case of $f(0) \neq 1$. If $f(0) \neq 1$, then we consider

$$
\tilde{f}(z)=\frac{f(z)-i \Im f(0)}{\Re f(0)} .
$$

By calculation, $\Re \tilde{f}(z)=\frac{\Re f(z)}{\Re f(0)}>0, \tilde{f}(0)=1$. Hence based on the result of the case above, we have

$$
\lim _{r \rightarrow 1}(1-r)^{n+1}\left|\tilde{f}^{(n)}(z)\right|=\lim _{r \rightarrow 1} 2 n ! \frac{1-r}{1+r} \Re \tilde{f}(z) .
$$

On the other hand, $\tilde{f}^{(n)}(z)=\frac{f^{(n)}(z)}{\Re f(0)}$. So we can change (3.1) to

$$
\lim _{r \rightarrow 1}(1-r)^{n+1}\left|\frac{f^{(n)}(z)}{\Re f(0)}\right|=\lim _{r \rightarrow 1} 2 n ! \frac{1-r}{1+r} \frac{\Re f(z)}{\Re f(0)},
$$

namely,

$$
\lim _{r \rightarrow 1}(1-r)^{n+1}\left|f^{(n)}(z)\right|=\lim _{r \rightarrow 1} 2 n ! \frac{1-r}{1+r} \Re f(z) .
$$

This completes the proof. 


\section{REFERENCES}

1. B. Maccluer, K. Stroethoff and R. Zhao, Generalized Schwarz-Pick estimates, Proc. A. M. S, 131 (2002), 593-599. MR1933351 (2003g:30038)

2. P. Ghatage and DeChao Zheng, Hyperbolic derivatives and Generalized Schwarz-Pick estimates, Proc. A. M. S, 132 (2004), 3309-3318. MR2073307 (2005e:30039)

3. P. L. Duren, Univalent functions, Springer (2001). MR708494 (85j:30034)

4. H. Boas, And D. Khavinson, Bohr's power series theorem in several variables, Proc. Amer. Math. Soc. 125 (1997), no. 10, 2975-2979. MR1443371 (98i:32002)

5. Y. Pan, and S. Liao, On the derivative of bounded analytic functions, Journal of Jiangxi Normal University (1984) 1 21-24.

General Study Program, Jinling Institute of Technology, Nanjing, People's RepubLIC OF CHINA

E-mail address: dymdsy@163.com

School of Mathematics and Informatics, Jiangxi Normal University, Nanchang, People's Republic of China; and Department of Mathematical Sciences, Indiana University - Purdue University Fort Wayne, Fort Wayne, Indiana 46805-1499

E-mail address: pan@ipfw.edu 